\section{MS24-P2 Monitoring packing changes in planar molecules}

Dean S. Keeble ${ }^{1}$, Luke A. Rochford ${ }^{2}$, Helen Y. Playford ${ }^{3}$, Matt G. Tucker $^{1,3}$

1. Diamond Light Source, Harwell Science and Innovation Campus, UK.

2. Department of Chemistry, University of Warwick, UK.

3. ISIS Facility, Rutherford Appleton Laboratory, Harwell Science and Innovation Campus, UK

\section{email: dean.keeble@diamond.ac.uk}

The way that small molecules pack together can have substantial ramifications on observed physical properties, optical adsorption, and device characteristics, even if the molecular structure is identical. Small pi-conjugated molecular semiconductors such as phthalocyanines, subphthalocyanines, and squaraines demonstrate great potential in organic photovoltaics, field effect transistors and light emitting diodes. Their properties are readily tuneable due to their ability to accommodate a variety of metal centres, and structures can be further modified by substitution of outer-skeleton hydrogen atoms for a huge number of other atoms or small chemical moieties. However, whilst the effects of these substitutions on physical properties are measured routinely, it is much more uncommon to study the underlying influence on intra- and inter-molecular bonding. Since many physical properties depend critically on chemical bonding, increased understanding of the influence of substitution could pave the way for the targeted design of new materials. We will present a pair distribution function (PDF) study of some exemplar molecular semiconductors, in order to demonstrate the ability of the PDF technique to directly study how metal and hydrogen substitution are structurally accommodated in these systems. In particular, we will look at how it is possible to observe changes in molecular packing using the PDF.

Keywords: pair distribution function, organic semiconductors, molecular packing, short-range order
MS24-P3 Phase transitions in $\mathrm{PbZr}_{1-x} \mathrm{Ti}_{x} \mathrm{O}_{3}$ with low Ti concentrations studied by $\mathrm{X}$-ray scattering

Daria A. Andronikova ${ }^{1,2}$, Alexei Bosak ${ }^{3}$, Yurii Bronwald ${ }^{1,2}$, Roman Burkovsky 2,3, Dmitry Chernyshov ${ }^{2,3}$, N. G. Leontyev ${ }^{4}$, I.N. Leontyev ${ }^{5}$, Sergey Vakhrushev ${ }^{1,2}$

1. Ioffe Institute, St. Petersburg, Russia

2. St.Petersburg Polytechnical University, St. Petersburg, Russia

3. ESRF, Grenoble, France

4. Azov-Black Sea State AgroEngineering Academy, Russia

5. Southern Federal University, Russia

email: andronikova.daria@gmail.com

$\mathrm{PbZr}_{1-\mathrm{x}} \mathrm{Ti}_{\mathrm{x}} \mathrm{O}_{3}(\mathrm{PZT})$ is a solid solution of lead titanates $\mathrm{PbTiO}_{3}$ and lead zirconates $\mathrm{PbZrO}_{3}$. $\mathrm{PZT}$ is the famous and the most practically used ferroelectric. The reason of this popularity is that PZT possesses exceptionally good piezoelectric properties, has the high value of dielectric constant and the high phase transition's temperature. Phase diagram of PZT demonstrates complexity. The area of our study is located at region of low concentration of Ti. There are two phases, namely ferroelectric and antiferroelectric one. Paraelectric-ferroelectric phase transition is accompanied by the appearance of the superlattice M-type reflections $1 / 2(\mathrm{~h} \quad \mathrm{k} \quad 0)$. Electron diffraction studies of ferroelectric phase have revealed the existence of the M-reflections splitting for PZT with high concentration of $\mathrm{Zr}$ [1]. At the moment corresponding structure modulation is not understood completely.

PZT single crystals X-ray diffraction at $\mathrm{Ti}$ concentrations $0,7 \%, 1,5 \%$ and $3,3 \%$ has been studied on Swiss-Norwegian beamline of European synchrotron radiation source. Diffraction measurements revealed the appearance of strong anisotropic diffuse scattering around Bragg spots. The splitting of M-superstructure was observed. The details are presented in the poster.

[1] J Ricote, D L Corker, R W Whatmore, A M Glazer, J Dec and K Roleder, Phys.: Condens. Matter 10, 1767-1786 (1998)

Keywords: phase transition, lead zirconate, X-ray scattering, diffuse scattering, antiferroelectric 